\title{
Integation Methods of Model-Free Features for 3D Tracking
}

\author{
Ville Kyrki and Kerstin Schmock \\ Lappeenranta University of Technology, \\ Laboratory of Information Processing, \\ P.O.Box 20, 53851 Lappeenranta, Finland \\ kyrki@lut.fi
}

\begin{abstract}
A number of approaches for 3D pose tracking have been recently introduced, most of them utilizing an edge (wireframe) model of the target. However, the use of an edge model has significant problems in complex scenes due to background, occlusions, and multiple responses. Integration of model-free information has been recently proposed to decrease these problems.

In this paper, we propose two integration methods for model-free point features to enhance the robustness and to increase the performance of real-time model-based tracking. The relative pose change between frames is estimated using an optimization approach. This allows the pose change to be integrated very efficiently in a Kalman filter. Our first approach estimates the pose change in a least squares sense while the second one uses M-estimators to decrease the effect of outliers. Experiments are presented which demonstrate that the approaches are superior in performance to earlier approaches.
\end{abstract}

\section{Introduction}

Real-time tracking of 3D pose is today one of the most important building blocks of computer vision systems in several application areas including augmented reality [1], activity interpretation [2], and robotic manipulation [3]. There is a multitude of approaches as most methods have been designed for a particular application. An important reason for this is that the visual characteristics are different in different applications. For example, some methods are suitable for textured objects $[3,1]$ while others are better suited for objects of uniform color $[4,2]$.

In 3D tracking, a 3D geometric model is usually available. Several successful systems for real-time pose tracking using such models have been proposed $[4,5]$. However, the model represents just the structure of the object using planes and lines, without the knowledge of surface texture. Because the object is tracked using the edge information, such systems are mainly suited to tracking of objects without texture in a relatively simple background. Otherwise the edges of the object are easily confused with background edges and object texture. 
Robustness of tracking can be increased by using multiple cues. Recently, it has been proposed to use automatically generated model-free point features to avoid the problems of the model-based tracking[6]. Thus, several different types of features are used to compensate for each others' weaknesses. A Kalman filter framework can be used in integrating the image-plane point measurements with the model-based 3D pose.

In this paper, we continue examining the integration approach. We present two new integration approaches which aim to increase the robustness and performance. The approaches are based on finding the relative pose change between frames by minimizing a sum of squared errors of the image point measurements. The second approach aims to further increase the robustness by decreasing the effect of possible outliers by using a robust M-estimator. We also experimentally compare the computational complexities and the accuracies of the new approaches to the existing approach.

Section 2 presents components of the tracking system including both modelbased and model-free trackers. In Sec. 3, we present the Kalman filter model of integration, along with the two new approaches. Experimental evaluation is described in Sec. 4. Finally, in Sec. 5, a short summary and conclusions are given.

\section{Tracking in 2D and 3D}

The tracking approaches are now reviewed independently for model-based and model free cues. A unit focal length is assumed below, i.e., the intrinsic camera calibration has been performed in advance. A more complete description can be found in $[6]$.

\subsection{Model-Based 3D Pose Tracking}

The model-based system consists of two main components. First, the initialization part, which is only used to find the pose in the first frame, and second, the tracking part, which is run real-time. The tracked objects are modeled using points, lines, and polygons defined in 3D. An example model can be seen in Fig. 1.

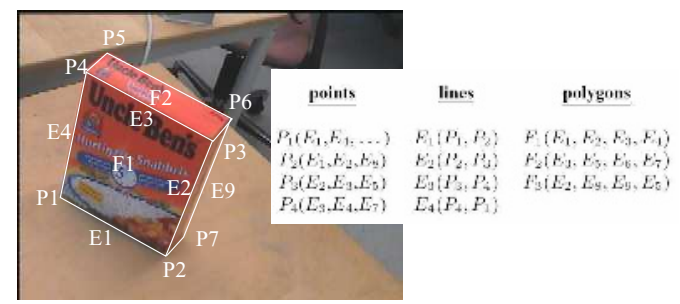

Fig. 1. Object representation using points, lines, and polygons 
In the initialization phase, wide baseline matching is performed in order to initialize the object in a general setting. SIFT features proposed in [7] are extracted off-line from images with the known object pose for each image. During the initialization, SIFT features are extracted from an image and matched with he ones extracted off-line. This is illustrated in Fig. 2. The view for which the number of matches is maximal is then used to estimate the pose of the object. For planar objects, planar homography estimation and decomposition with robust outlier rejection (RANSAC) is used for the pose estimation. This approach is used because for each point it is enough to know that it lies on the same plane, and the exact $3 \mathrm{D}$ position is not needed.

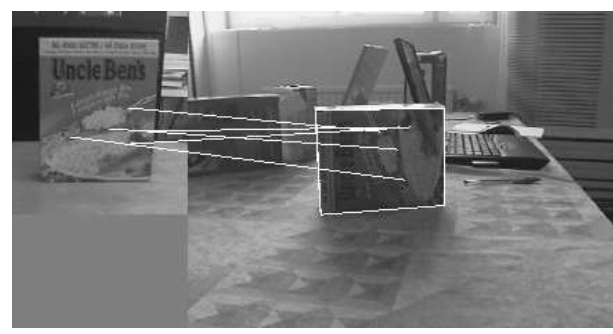

Fig. 2. Initialization using SIFT point features

The system for model-based pose tracking is based on ideas proposed by Drummond and Cipolla [4]. The basic idea is to estimate the normal flow for a set of points along the edges of the object. The points are distributed with equal image plane intervals along the edges. Lie algebra and Lie group formalism is used to represent the motion of a rigid body such that a six-dimensional Lie group represents the six degrees of freedom of the rigid body motion. The normal flow estimates are then used to find the least squares approximator for the motion by considering the generators of the group at identity. Thus, the motion estimates are good if the change of pose remains small between two frames. The method is very efficient and reasonably accurate if the normal flow estimates are precise.

\subsection{Model-Free 2D Point Tracking}

In addition to the $3 \mathrm{D}$ pose, individual interest points are located and tracked in the image. The automatic initialization extracts interest points using Harris corner detection [8]. The current pose estimate is used to only initialize interest points on the frontal plane of the tracked object. New interest points are reinitialized once every 10 frames to guarantee that enough good ones are available at all times. When a new interest point is chosen, its local neighborhood is stored.

The points are tracked in subsequent images by minimizing the sum of squared differences in the RGB values in the local neighborhood. This simple approach is computationally light while still allowing tracking over short time intervals. It should be noted that in contrast to some other proposed methods, 
the same interest point does not need to be tracked through the whole sequence. The tracked points are rejected on two conditions: i) if the sum of squared differences grows above a set threshold, or ii) if the point moves out of the frontal plane of the object.

\section{Kalman Filter Integration}

The two types of measurements are integrated in an iterated extended Kalman filter framework. IEKF estimates the state $\mathbf{x}$ of a system by

$$
\mathbf{x}_{i+1}=f\left(\mathbf{x}_{i}\right)+\mathbf{w}_{i} \quad \mathbf{y}_{i}=h\left(\mathbf{x}_{i}\right)+\mathbf{v}_{i}
$$

where $f(\mathbf{x})$ is the system model which describes the time dependencies of the system, $h(\mathbf{x})$ is the measurement model which links the internal state to measurable quantities, $\mathbf{y}$ is the measurement, and $\mathbf{w}$ and $\mathbf{v}$ are the system noise components modeled as Gaussian random variables. The IEKF estimation consists of two steps: First, the evolution of the system is predicted using the system model. Second, the state is updated using the measurements. For a more thorough description of IEKF, see [6].

The system state consists of the $3 \mathrm{D}$ pose of the tracked object,

$\mathbf{x}=\left(T_{X}, T_{Y}, T_{Z}, \phi_{x}, \phi_{y}, \phi_{z}\right)^{T}$. In this paper, we will concentrate on examining the methods for integrating model free features and thus only a zeroth order moving object system model is considered. The system is thus predicted to remain in the previous state, and the prediction covariance assumes that the object is rotating around its own origin. See [6] for an additional moving camera model.

For integrating the point measurements, three approaches are considered: 1) directly integrating the measurements in the Kalman filter using a suitable measurement model, introduced in [6]; 2) finding the optimal inter-frame transformation using the points, and 3) using M-estimators to find more robust estimates of the pose change. The two latter ones have a linear measurement model. The three approaches have different computational complexities and error characteristics. In all of the approaches, the following simplification is made in contrast to traditional structure-from-motion: Every time a point feature is initialized, its 3D coordinates in the object frame are calculated as the intersection of the line going through the point and camera origin, and the plane defined by the model and the current pose estimate. Thus, the depth and its error are not included in the state, but the associated uncertainty is modeled as part of the measurement noise. This choice allows using the Kalman filter in real-time with a large number of tracked points.

In all of the three approaches, the model-based measurements directly estimate the pose and thus: $h_{0}(\mathbf{x})=\mathbf{x}$. The measurement errors are assumed to be independent of orientation, therefore the measurement covariance matrix $\mathbf{S}_{0}$ can be written

$$
\mathbf{S}_{0}=\left(\begin{array}{cc}
\sigma_{t}^{2} \mathbf{I}_{3} & \mathbf{0} \\
\mathbf{0} & \sigma_{\phi}^{2} \mathbf{I}_{3}
\end{array}\right)
$$

where $\sigma_{t}^{2}$ and $\sigma_{p} h i^{2}$ are the variances of translation and rotation measurements. 


\subsection{Direct Integration}

In the direct integration approach, the measurement function is the perspective projection of the known $3 \mathrm{D}$ point position $\mathbf{q}_{j}$ :

$$
\begin{aligned}
\left(X_{j} Y_{j} Z_{j}\right)^{T} & =R\left(\phi_{x}, \phi_{y}, \phi_{z}\right) \mathbf{q}_{j}+t(\mathbf{x}) \\
h_{j}(\mathbf{x}) & =\left(X_{j} / Z_{j} Y_{j} / Z_{j}\right)^{T}+\mathbf{v},
\end{aligned}
$$

where $R(\cdot)$ is the rotation matrix taking into account the current rotation estimate described by the state, and $t(\mathbf{x})$ is the translation component of the state. The same form of the function is used for each of the points.

The measurement errors are assumed to be independent with respect to the coordinate axes and thus the measurement covariance matrix can be written $\mathbf{S}_{1}=\sigma_{i}^{2} \mathbf{I}$ where $\sigma_{i}^{2}$ is the image measurement variance. The gradient of the measurement function is calculated analytically from the perspective projection (3) but not shown here for the sake of brevity.

\subsection{Basic Optimization}

The basic optimization approach minimizes the sum of squared errors criterion between image measurements and the initialized 3D points. Thus, the minimized quantity is the $3 \mathrm{D}-2 \mathrm{D}$ projection error $d(\cdot)$

$$
d=\sum_{j} e_{j}^{2} \quad e_{j}=\sqrt{\left(x_{j}-\frac{X_{j}}{Z_{j}}\right)^{2}+\left(y_{j}-\frac{Y_{j}}{Z_{j}}\right)^{2}}
$$

where $\left(x_{j}, y_{j}\right)$ is the measured position of a point, and $\left(X_{j}, Y_{j}, Z_{j}\right)$ are its coordinates in the current camera frame, from Eq. 3. Note that the camera frame coordinates then depend on the current estimated pose.

The optimization is performed using the Polak-Ribiere variant of the conjugate gradient method [9]. Initial search direction is to the negative error function gradient, $\mathbf{g}_{0}=-\nabla d\left(\mathbf{x}_{0}\right)$. The search direction for successive iterations is determined using

$$
\mathbf{g}_{k+1}=-\nabla d\left(\mathbf{x}_{k+1}\right)+\gamma_{k} \mathbf{g}_{k}
$$

where

$$
\gamma_{k}=\frac{\left(\nabla d\left(\mathbf{x}_{k+1}\right)-\nabla d(\mathbf{x})\right) \cdot \nabla d\left(\mathbf{x}_{k+1}\right)}{\nabla d\left(\mathbf{x}_{k}\right) \cdot \nabla d\left(\mathbf{x}_{k}\right)}
$$

Each line minimization is performed by first bracketing the minimum using golden section bracketing. Then the search for minimum is performed by Brent's method which uses parabolic interpolation and golden section search [10].

\subsection{M-Estimator Based Optimization}

It is well known that a least squares optimization is vulnerable to outliers. To increase the robustness and decrease the effect of outliers, we also present a 
method, which employs M-estimators, introduced by Huber [11], for outlier rejection.

In M-estimators, the squared residual is replaced with another function of the residual. The problem can be solved as iteratively reweighted least squares, such that for each measurement, a weight function is determined, standard least squares is performed, and these two steps are iterated. We use the Tukey weight function [12]. Thus, we repeatedly solve the problem

$$
\min \sum_{j} w_{T U K}\left(e_{j}\right) e_{j}^{2}
$$

where $e_{j}$ is the residual for point $j$ from (4) and $w_{T U K}$ is the Tukey weight function

$$
w_{T U K}(x)= \begin{cases}\left(1-(x / c)^{2}\right)^{2} & \text { if }|x| \leq c \\ 0 & \text { if }|x|>c\end{cases}
$$

where $c$ is a parameter of the estimator. Tukey weight was chosen because it has been successfully used in similar contexts, e.g., [1]. Each individual least squares minimization is performed using the conjugate gradient method described above. We found out experimentally that two least squares iterations solve the problem to the accuracy of the measurements, and thus the number of iterartions was fixed to two. This is mainly because the inter-frame times are short and motions small, and therefore the initial point of the optimization is already very close to the optimum.

\section{Experimental Comparison}

Experiments are now presented to inspect the two new proposed methods. In particular, three characteristics are investigated: i) accuracy, ii) performance, and iii) robustness. The experiments were performed on two recorded sequences to allow repeated tests. Both sequences had a different target object and can be seen in Fig. 3. The lengths of the sequences are 173 (Sequence 1) and 157 seconds (Sequence 2). The sequences were recorded by moving a camera mounted on a

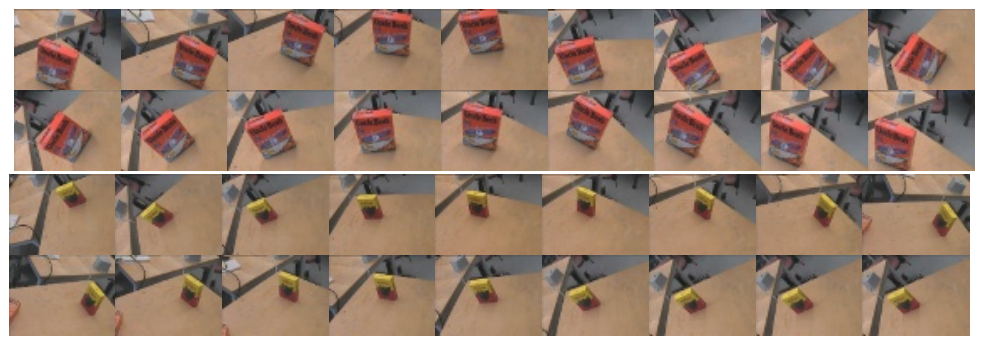

Fig. 3. Test sequences: Sequence 1 (top); Sequence 2 (bottom) 
robot arm. Ground truth was generated by recording the robot trajectory and determining the hand-eye calibration by the method of Tsai and Lenz [13]. The camera was only coarsely calibrated, the optical axis was assumed to coincide with the center pixel and the manufacturer given focal length was used. A typical number of tracked points was 25 for Sequence 1 and 8 for Sequence 2, but the number changed significantly during both sequences.

\subsection{Accuracy}

Tracking accuracy was studied for both sequences and the three integration methods described in Sec. 3. Figure 4 shows the error magnitudes for Sequence 1.
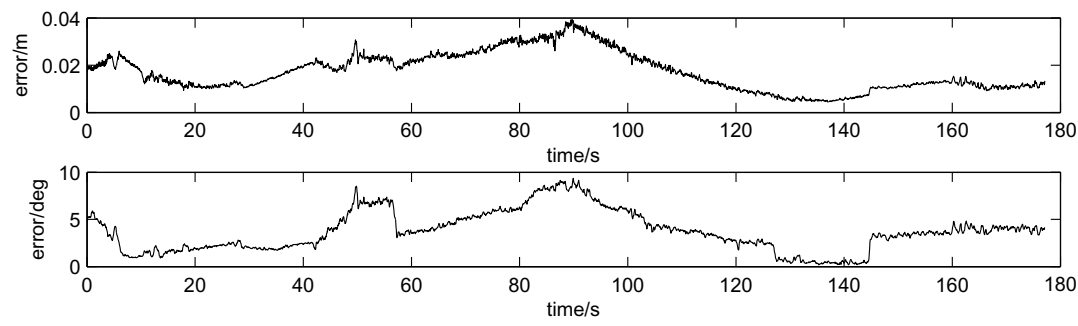

(a)
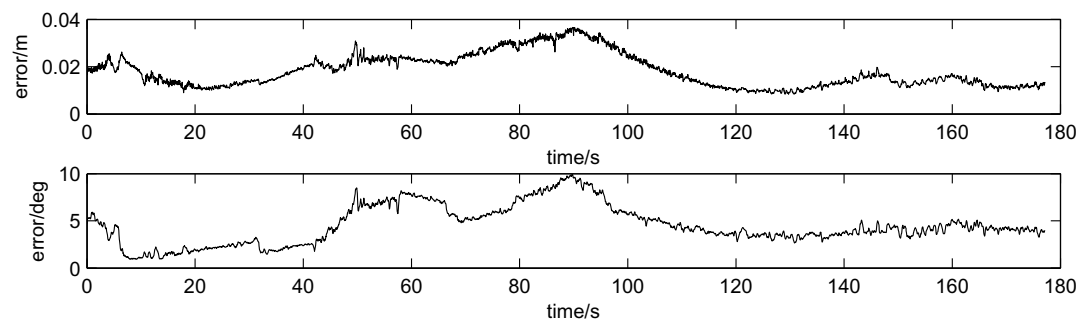

(b)
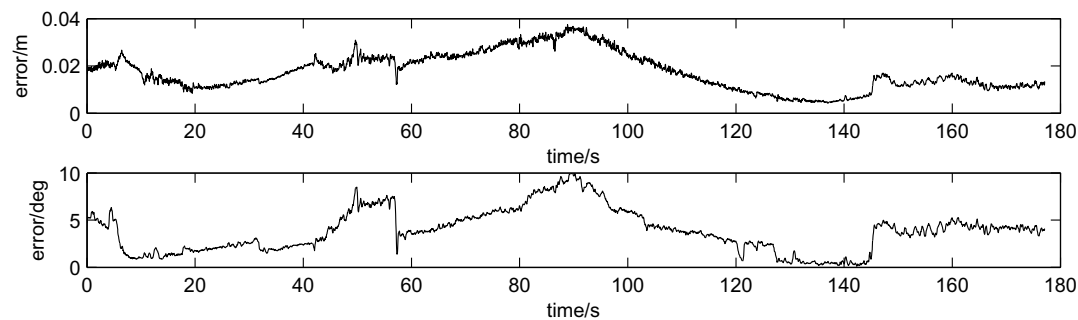

(c)

Fig. 4. Tracking in Sequence 1: (a) direct integration; (b) basic optimization; (c) Mestimator optimization 
Table 1. Average frame times and frame rates

\begin{tabular}{|l|r|r|}
\hline & Avg. frame time $(\mathrm{ms})$ & Frame rate $(\mathrm{Hz})$ \\
\hline Direct integration & 61.1 & 16.3 \\
\hline Basic optimization & 11.6 & 86.0 \\
\hline M-estimator opt. & 13.1 & 76.2 \\
\hline
\end{tabular}

All integration methods have similar behavior. Direct integration having slightly lower average error of $1.7 \mathrm{~cm}$ in translation and 3.8 degrees in rotation compared to $1.8 \mathrm{~cm}$ and 4.5 degrees for basic optimization and $1.8 \mathrm{~cm}$ and 3.9 degrees for M-estimator optimization. It can be seen that the basic optimization is slightly inferior to the other two approaches, resulting from the fact that tracked points include some measurement errors, which have unnecessarily large contribution to the trajectory tracked.

For Sequence 2, there were no significant differences, and the average errors were $6.5 \mathrm{~cm}$ and 12.2 degrees for both direct integration and basic optimization, and $6.5 \mathrm{~cm}$ and 12.0 degrees for M-estimator optimization.

\subsection{Performance}

The performance was evaluated by measuring the processor times for the tracking using the three different integration approaches. The total processor time spent during whole Sequence 1 was measured, and then the average frame time and frame rate were calculated. Only Sequence 1 was used because it is longer of the two, in order to minimize the effect of initialization to the computational cost. The tests were run on a $1.7 \mathrm{GHz}$ Pentium M laptop. The results are shown in Table 1.

It is evident from Table 1 that the new methods presented in this paper significantly improve the performance. Basic optimization is slightly faster of the two new methods, but both are clearly suitable for real-time use. The main reason for the improved performance is that the Kalman filter measurements have fewer dimensions. In the direct integration, each tracked point generates two measurements ( $x$ - and $y$-coordinates) while in the optimization approaches the model-free measurement is the 6-dimensional pose. For this reason, the Kalman filter update operates on significantly smaller matrices, In addition, the pose measurement is linear to the filter state, and thus no measurement gradient needs to be computed.

The actual runtimes were somewhat larger than the ones above, because the sequences were stored on a hard drive and the disk $\mathrm{I} / \mathrm{O}$ time is not included in the times given above. For all three methods, the delay of disk I/O was found to be equal, so the results can be compared.

\subsection{Robustness}

The robustness of the two optimization based pose tracking methods was inspected by ignoring totally the model-based cue. The trajectories without model- 

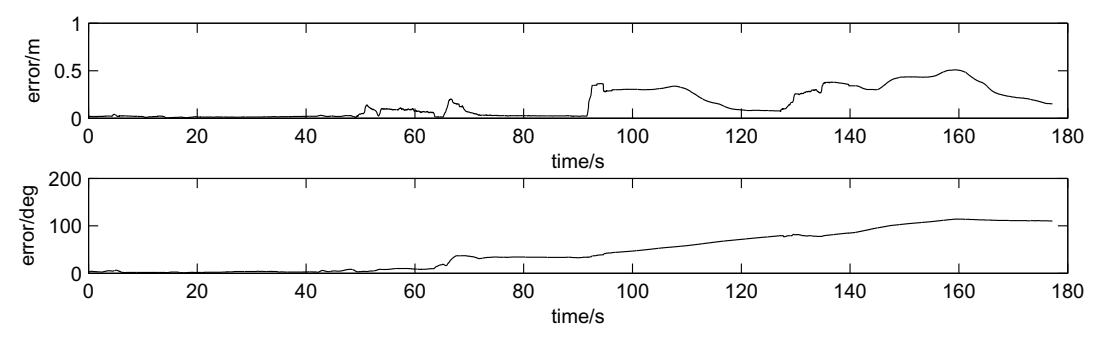

(a)
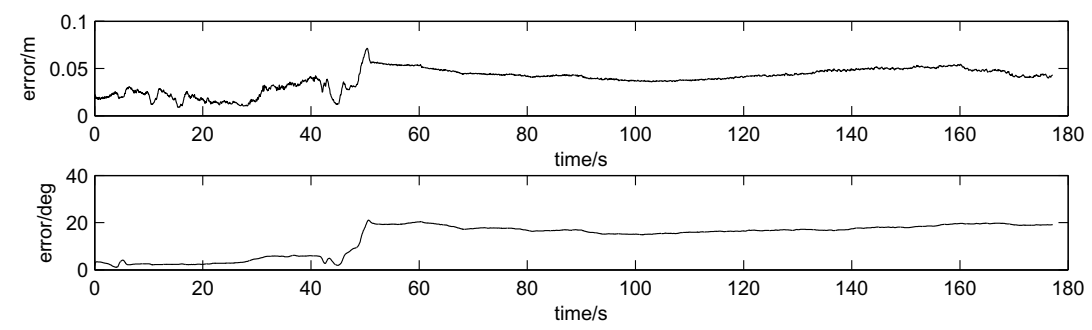

(b)

Fig. 5. Drift in model-free tracking: (a) Basic optimization; (b) M-estimator optimization

based tracking are shown in Fig. 5. Note also the different scaling in the vertical axis in the two graphs. In this case, the tracking is prone to drift. It can be seen in the figure that both methods start to drift after 50 seconds. However, the basic optimization approach soon loses the object position entirely, while the Mestimator approach is capable to keep approximate track of the object position through the whole sequence. The small errors in the translation also indicate that the object position in the image can be tracked. M-estimator approach performs significantly better with $3.9 \mathrm{~cm}$ average translation error and 13.8 degree rotation error compared to both direct integration $(25 \mathrm{~cm} / 25$ degrees $)$ and basic optimization (15 cm/46 degrees). This is explained by the fact that because model-based correction is not made, some tracked points fall outside the object. Therefore the basic optimization approach fails because of the outliers, while the M-estimator approach rejects the outliers.

\section{Conclusion}

Pose tracking systems based on edge models have significant problems in complex scenes due to background, occlusions, and multiple responses. To cope with the problems above, we have presented in this paper two methods of integrating 
information from model-free point features in 3D tracking. Both methods use a Kalman filter framework for integrating the features.

Experimental results present an evaluation of the methods with a comparison to the earlier direct integration method. The accuracy of the new methods was comparable to the earlier one. However, computationally the new methods were found to outperform the old one significantly, making video rate operation possible. Finally, the robustness of the methods was evaluated in model-free tracking, which showed that the optimization using M-estimators was clearly more robust against outlier measurements.

Future work will consider tracking more complex geometric shapes, not consisting of planar patches. Also, to improve the tracking against occlusions, we plan to improve the rejection mechanism of the image plane trackers by discarding trackers incompatible with the detected motion.

\section{References}

1. Vacchetti, L., Lepetit, V., Fua, P.: Stable real-time 3D tracking using online and offline information. IEEE Trans PAMI 26 (2004) 1385-1391

2. Vincze, M., Ayromlou, M., Ponweiser, M., Zillich, M.: Edge projected integration of image and model cues for robust model-based object tracking. Int J of Robotics Research (2001)

3. Taylor, G., Kleeman, L.: Fusion of multimodal visual cues for model-based object tracking. In: Australiasian Conf. on Robotics and Automation, Brisbane, Australia (2003)

4. Drummond, T., Cipolla, R.: Real-time visual tracking of complex structures. IEEE Trans. PAMI 24 (2002) 932-946

5. Wunsch, P., Hirzinger, G.: Real-time visual tracking of 3-d objects with dynamic handling of occlusion. In: IEEE Int. Conf. on Robotics and Automation, ICRA'97, Albuquerque, New Mexico, USA (1997) 2868-2873

6. Kyrki, V., Kragic, D.: Integration of model-based and model-free cues for visual object tracking in 3D. In: Int Conf on Robotics and Automation, ICRA'05. (2005)

7. Lowe, D.: Distinctive image features from scale-invariant keypoints. Int. J. Comp. Vis. 60 (2004) 91-110

8. Harris, C.J., Stephens, M.: A combined corner and edge detector. In: Proc. 4th Alvey Vision Conference, Manchester, UK (1988)

9. Press, W.H., Teukolsky, S.A., Vetterling, W.T., Flannery, B.P.: Numerical Recipes in $\mathrm{C}++$. Cambridge University Press (2002)

10. Brent, R.P.: Algorithms for Minimization without Derivatives. Prentice-Hall (1973)

11. Huber, P.J.: Robust estimation of a location parameter. Annals of Mathematical Statistics 35 (1964) 73-101

12. Huber, P.J.: Robust Statistics. Wiley (1981)

13. Tsai, R., Lenz, R.K.: A new technique for fully autonomous and efficient 3D robotics hand/eye calibration. IEEE Trans. Robotics and Autom. 5 (1989) 345358 